\title{
ON THE GROUPS OF INERTIA OF SMOOTH MANIFOLDS ${ }^{1}$
}

\author{
ADIL G. NAOUM
}

Abstract. In this paper we study sufficient conditions for a manifold $M^{n}$ to have $I\left(M^{n}\right)=\{0\}$. We also prove that if $M^{n}$ is a smooth manifold of dimension $n, n \equiv 2(\bmod 8)$, with $w_{2}\left(M^{n}\right) \neq 0$, then $I\left(M^{n}\right) \neq 0$.

Introduction. In [8] Kosinski defined, for a smooth oriented closed manifold $M^{n}$, of $I\left(M^{n}\right)$, to be the subgroup of $\theta^{n}$ consisting of all homotopy spheres $\Sigma^{n}$ for which there exists an orientation preserving diffeomorphism from $M^{n}$ to $M^{n} \# \Sigma^{n}$. $\theta^{n}$ is the group of homotopy spheres defined by Kervaire and Milnor in [6]. \# stands for connected sum of two manifolds. $I\left(M^{n}\right)$ is the group of inertia of $M^{n}$.

In $\S 1$, we give some sufficient conditions for a manifold $M^{n}$ to have $I\left(M^{n}\right)=\{0\}$. In $\S 2$, we study smooth manifolds of dimension $n, n \equiv 2$ (mod 8) and with $\omega_{2}(M) \neq 0$ and show that $I\left(M^{n}\right)$ for these manifolds does not vanish. All manifolds considered in this paper are oriented $C^{\infty}$ manifolds, unless otherwise stated.

1. Manifolds with zero groups of inertia. In this section we give some sufficient conditions for a manifold $M^{n}$ in order to have $I\left(M^{n}\right)=0$.

Definition. We say that a manifold $M^{n}$ satisfies property (S) if $H_{i}\left(M^{n}, Z\right)$ is a cyclic group for all $i \geqq 0$.

THEOREM. Let $M^{n}$ be a smooth closed manifold of dimension $n \geqq 7$ satisfying property (S). Then if $M^{n}$ imbeds smoothly in $S^{n+1}$ and bounds a simply connected region in $S^{n+1}$, then $I\left(M^{n}\right)=0$.

Proof. Let $M^{n} \subseteq S^{n+1} . M^{n}$ separates $S^{n+1}$ into two components $A, B$ such that

$$
A \cup B=S^{n+1} ; \quad A \cap B=M^{n}=b A=b B .
$$

$A, B$ are smooth manifolds with boundary $M^{n}$.

Received by the editors July 27, 1972 and, in revised form, November 22, 1972 and January 12, 1973.

AMS (MOS) subject classifications (1970). Primary 57D55.

Key words and phrases. Smooth manifold, group of inertia, manifolds with zero group of inertia.

${ }^{1}$ This paper constitutes a portion of the author's Ph.D. dissertation submitted to Courant Institute of Mathematical Sciences, NYU in 1968 and written under the supervision of Professor M. Kervaire to whom the author expresses his deep gratitude.

(C) American Mathematical Society 1973 
Let $\Sigma^{n}$ be a homotopy sphere that represents an element in $I\left(M^{n}\right)$, then there exists an orientation preserving differomorphism

$$
\phi: M^{n} \# \Sigma^{n} \rightarrow M^{n} \text {. }
$$

Let $B^{\prime}=B \#\left(\sum^{n} \times I\right)$ be the connected sum of $B$ and $\Sigma^{n} \times I$ along $b B$ and $\Sigma^{n} \times(0)$. Let $W^{n+1}$ be the manifold obtained from $A$ and $B^{\prime}=B \#\left(\sum^{n} \times I\right)$ by pasting $M^{n}$ and $M^{n} \# \Sigma^{n}$ via the diffeomorphism $\phi . b W=\Sigma^{n}$. It follows from van Kampen's theorem that $W$ is simply connected.

We consider the Mayer-Vietoris homology sequences with integer coefficients for $S^{n+1}$ and for $W^{n+1}$. For $i+1<n+1$,

$$
\begin{aligned}
0= & H_{i+1}\left(S^{n+1}\right) \rightarrow H_{i}\left(M^{n}\right) \stackrel{i_{A} \oplus\left(-i_{B}\right)}{\longrightarrow} H_{i}(A) \oplus H_{i}(B) \rightarrow H_{i}\left(S^{n+1}\right) \\
& \rightarrow H_{i+1}(W) \rightarrow H_{i}\left(M^{n}\right) \stackrel{i_{A} \oplus\left(-i_{B^{\circ}} \phi^{-1)}\right.}{\longrightarrow} H_{i}(A) \oplus H_{i}\left(B^{\prime}\right) \rightarrow H_{i}\left(W^{n+1}\right) \rightarrow .
\end{aligned}
$$

We have

$$
H_{i}\left(M^{n}\right) \stackrel{i_{A} \oplus\left(-i_{B}\right)}{\longrightarrow} H_{i}(A) \oplus H_{i}(B)
$$

is an isomorphism and ker $i_{A} \cap \operatorname{ker} i_{B}=0$.

Since $H_{i}\left(M^{n}\right)$ is cyclic, we have both $\operatorname{ker} i_{A}$ and ker $i_{B}$ are cyclic groups of different orders. On the other hand we can identify the homology groups of $M^{n}$ with those of $M^{n} \# \Sigma^{n}$ and consider $\phi_{*}^{-1}$ as an automorphism of $H_{i}\left(M^{n}\right)$ onto $H_{i}\left(M^{n} \# \Sigma^{n}\right)$ and this automorphism leaves ker $i_{B}$ invariant. Therefore $\operatorname{ker} i_{A} \cap \operatorname{ker}\left(i_{B^{\prime}} \circ \phi_{*}^{-1}\right)=0$; and hence $i_{A}+\left(-i_{B^{\prime}} \circ \phi_{*}^{-1}\right)$ is injective. We show $i_{A}+\left(-i_{B^{\prime}} \circ \phi_{*}^{-1}\right)$ is surjective. Let $x \in H_{i}(A) \oplus H_{i}\left(B^{\prime}\right)$. $x$ corresponds to an element, call it $x$ again, in $H_{i}(A) \oplus H_{i}(B)$. Hence $x=a+b, \quad a \in H_{i}(A), \quad b \in H_{i}(B)$. Put $y=i_{A}^{-1}(a)+\left(-i_{B}^{-1} b\right) ; \quad y^{\prime}=i_{A}^{-1}(a)+$ $\left(-\phi_{*} i_{B}^{-1} b\right)$. Hence $\left[i_{A}+\left(-i_{B^{\prime}} \circ \phi_{*}^{-1}\right)\right]\left(y^{\prime}\right)=x$. Thus $i_{A}+\left(-i_{B^{\prime}} \circ \phi_{*}^{-1}\right)$ is an isomorphism for all $i<n$.

Therefore, $H_{i}(W)=0,1 \leqq i \leqq n$; and since $\Pi_{1} W=0, W$ is a contractible manifold and hence $\Sigma^{n}=b W$ is diffeomorphic to $S^{n}$, i.e. it represents the zero element in $\theta^{n}[6]$. This proves that $I\left(M^{n}\right)=0$.

COROLlaRY. Let $M^{n}$ be a smooth manifold satisfying property (S). Let $T$ be the total space of the normal sphere bundle of $M^{n}$ in $S^{n+k}$ for $k>n+1$. Then $I(T)=0$.

As an example of this situation, we have:

COROLlary. Let $T$ be the total space of the normal sphere bundle of $P_{n}(c)$, the complex projective space of dimension $n$, in $S^{n+k}, k>2 n+1$, then $I(T)=0$.

In [5] Steer and Brown proved that $I\left(V_{n}\right) \neq 0$, where $V_{n}$ is the Stiefel manifold of unit tangent vectors to $S^{n}, n$ odd, $n \neq 1,3,7$. In fact $I\left(V_{n}\right) \supseteq$ $\theta^{2 n-1}(\partial \pi)=$ the subgroup of $\theta^{2 n-1}$ consisting of those homotopy spheres 
that bound parallelizable manifolds. Since $S^{7}$ is parallelizable, $V_{7} \approx S^{7} \times S^{6}$ and by the Theorem, $I\left(V_{7}\right)=0$. We show next that this is a special case of a more general situation, namely:

COROLLARY. Let $M^{n}$ be a simply connected manifold satisfying property (S), and $M$ is parallelizable, then if $T^{2 n-1}$ is the total space of the tangent sphere bundle of $M^{n},\left(T^{2 n-1} \approx M^{n} \times S^{n-1}\right), I\left(T^{2 n-1}\right)=0$.

Proof. It is enough to show $T$ imbeds in $S^{2 n}$ since $T$ satisfies (S). Imbed $M^{n}$ in $S^{2 n}$. The obstruction to the triviality of the normal bundle of $M^{n} \subset S^{2 n}$ lies in $\operatorname{ker} J_{n} \cap \operatorname{ker} i_{n}$ where

$$
J_{n}: \Pi_{n-1}\left(S O_{n}\right) \rightarrow \Pi_{2 n-1}\left(S^{n}\right)
$$

is the $J$-homomorphism, and

$$
i_{n}: \Pi_{n-1}\left(S O_{n}\right) \rightarrow \Pi_{n-1}(S O)
$$

is the map induced by the inclusion of $S O_{n}$ in $S O$. But ker $J_{n} \cap$ ker $i_{n}=0$, which implies that the normal bundle of $M^{n} \subset S^{2 n}$ is trivial, and hence the tangent sphere bundle which is diffeomorphic to $M^{n} \times S^{n-1}$ imbeds in $S^{2 n}$.

We recall that the mod 2 semicharacteristic of an odd dimensional manifold $M^{2 n-1}$ is defined to be $\sum_{i=0}^{n-1} b_{i}$, where $b_{i}$ is the rank of the mod 2 homology group $H_{i}\left(M, Z_{2}\right)$.

TheOREM (ADAMS-Kervaire). Let $M$ be a closed $\Pi$-manifold.

(i) When $M$ is even dimensional, $M$ is parallelizable if and only if its Euler characteristic is zero.

(ii) When $M$ is odd dimensional, $M$ is parallelizable if and only if either its dimension is $1,3,7$, or its mod 2 semicharacteristic is even.

For a proof, see [7].

Using this theorem, we get

COROLlaRY. Let $M^{n}$ be a simply connected П-manifold satisfying property (S). If $n$ is even, assume the Euler characteristic is zero. If $n$ is odd, $n \neq 1,3,7$, assume the mod 2 semicharacteristic is zero. Then the group of inertia of the tangent sphere bundle of $M^{n}$ is zero.

COROLlary. The group of inertia of the tangent sphere bundle of $V_{n}$ is zero.

2. Manifolds with nonzero groups of inertia. In this section we give some sufficient conditions on a closed connected smooth manifold in order to have the group of inertia contain more than one element. Before stating the main theorem we recall some well-known facts.

Let $\xi$ be a universal spin $(8 k)$ bundle, let $D(\xi), S(\xi)$ be the total spaces 
of the associated disk and sphere bundles respectively. Put

$$
M(\operatorname{spin} 8 k)=D(\xi) / S(\xi)
$$

for the universal Thom space of $\xi$. It is known that

$$
\Pi_{n+8 k}(M \operatorname{spin} 8 k) \cong \Omega_{n}^{\text {spin }}
$$

where $\Omega_{n}^{\text {spin }}$ is the $n$th spin cobordism group. Moreover, for $n \not \equiv 0(\bmod 4)$, $\Omega_{n}^{\text {spin }}$ is finite. Let

$$
P_{n}: \theta^{n} \rightarrow \Omega_{n}^{\text {spin }}
$$

be the map which assigns to each element $\Sigma^{n} \in \theta^{n}$ its cobordism class in $\Omega_{n}^{\text {spin }}$. Let $K_{n}=$ kernel $P_{n}$.

The proof of the following proposition is in [2]; see also [9].

Proposition. For $r \equiv 1,2 \bmod 8, r>2$, the image of the homomorphism $P_{r}: \theta^{r} \rightarrow \Omega_{r}^{\text {spin }}$ is nontrivial.

Now we state the main theorem of this section.

THEOREM. Let $M^{n}$ be a closed 1-connected smooth manifold, $n \equiv 2$ $(\bmod 8), n>2$. Then $I\left(M^{n}\right) \subseteq K_{n}$ if and only if $\omega_{2}\left(M^{n}\right)=0$, the second Whitney class of $M^{n}$. In particular if $\omega_{2}\left(M^{n}\right) \neq 0, I\left(M^{n}\right) \neq 0$.

For the proof of the "if" half see [4] or [9]. Next we recall the theorem of J. F. Adams [1, Theorem 1.2].

Theorem (Adams). Suppose that $r \equiv 1$ or $2 \bmod 8$ and $r>0$. Then $\Pi_{r}^{s}=\lim _{n \rightarrow \infty} \Pi_{n+r}\left(S^{n}\right)$ contains an element $\mu_{r}$ of order 2 such that if $i_{*}$ : $\Pi_{r+8 k}\left(S^{k}\right) \rightarrow \Pi_{r+8 k}(M$ spin $8 k)$ is the homomorphism induced by inclusion, then $i_{*} \mu_{r} \neq 0$.

We now proceed to prove that the homotopy spheres represented by the $\mu_{n}$ lie in $I\left(M^{n}\right)$ for every 1 -connected manifold with $\omega_{2}\left(M^{n}\right) \neq 0, n \equiv 2 \bmod 8$. We start by recalling some results of Novikov [10].

Let $M^{n}$ be a closed manifold; $T\left(v_{M}\right)$ be the Thom space of the normal bundle of $M^{n} \subset S^{n+N}$ for $N$ large. There exists a natural map

$$
k: S^{\nu} \rightarrow T\left(v_{M}\right)
$$

induced by an inclusion of a fiber.

Let $M_{1}^{n}$ be a manifold tangentially equivalent to $M^{n}$. Put $B\left(M_{1}^{n}\right)=$ the set of all homotopy classes $\alpha \in \Pi_{n+. v}(T)$ for which there exists a representative $f_{\alpha}$

$$
f_{\alpha}: S^{n+N} \rightarrow T\left(v_{M}\right)
$$

such that $f_{\alpha}$ is transverse regular to $M \subset T\left(v_{M}\right), f_{\alpha}^{-1}(M)=M_{1}$ and $f_{\alpha} \mid M_{1}$ is the tangential equivalence between $M_{1}$ and $M$. 
For any homotopy sphere $\Sigma^{n}$, Kervaire and Milnor in [6] defined $P\left(\Sigma^{n}\right) \subseteq \Pi_{n}^{s}$ to be the set of all homotopy classes in $\Pi_{n}^{s}$ which can be obtained from all possible framings of $\Sigma^{n} \subseteq S^{n+N}$, using the ThomPontriagin construction. Taking $M=M_{1}$, we have

Lemma (Novikov) [10]. $B\left(M^{n} \# \Sigma^{n}\right) \supseteq B\left(M^{n}\right)+k_{*} P\left(\Sigma^{n}\right)$ where $k_{*}$ : $\Pi_{n+N}\left(S^{N}\right) \rightarrow \Pi_{n+N}(T)$ is the map induced from $k$.

COROLlaRY. Assume there exists an element $\gamma \in P\left(\Sigma^{n}\right)$ such that $k_{*} \gamma=0$, then there exists a homotopy sphere $\Sigma_{1}^{n} \in \theta^{n}(\partial \pi)$ such that $\Sigma^{n} \# \Sigma_{1}^{n} \in I\left(M^{n}\right)$.

Proof. Let $\alpha \in B\left(M^{n}\right)$ be a "normal invariant" of $M^{n}$. It follows from the assumption and the last lemma that $\alpha$ is also a normal invariant of $M^{n} \# \Sigma^{n}$. Now by a theorem of Browder-Novikov, if two manifolds correspond to the same normal invariant, they are diffeomorphic modulo $\theta^{n}(\partial \pi)[\mathbf{1 0}]$.

Lemma. Let $M^{n}$ be a 1-connected closed manifold, $n \equiv 2 \bmod 8, n>2$. Assume $\omega_{2}\left(M^{n}\right) \neq 0$. Let $T=T\left(v_{M}\right)$, the Thom space of the normal bundle of $M$ in $S^{n+N}$. Then $k_{*} \mu_{n}=0$, where the $\mu_{n}$ are Adams elements.

Proof. Since $\Pi_{1} M=\{1\}$, and $\omega_{2}\left(M^{n}\right) \neq 0$, there exists a sphere $S^{2}$ imbedded in $M^{n}$ with nontrivial normal bundle (in fact with a characteristic class $\alpha$, the nonzero element of $\left.\Pi_{1}\left(S O_{n-2}\right)=Z_{2}\right)$.

The normal bundle $v$ of $M^{n} \subset S^{n+N}$, when restricted to $S^{2}$, is just $E^{N-n+2} \alpha=$ the iterated suspension of $\alpha$. Put $\beta=E^{N+n+2} \alpha \in \Pi_{1}\left(S O_{N}\right)$. If $T_{0}$ is the Thom space of $\beta$, we have $T_{0} \subseteq T$. But, by the definition of the $J$-homomorphism we have

$$
T_{0}=S^{N} \cup_{J \beta} D^{N+2}, \quad J \beta \in \Pi_{N^{+}+1}\left(S^{N}\right)=I_{1}^{s} .
$$

Let $f: S^{N+n} \rightarrow S^{N}$ be a representative of $\mu_{n}$. Adams defined $\mu_{n}=\mu_{8 s+2}$ to be $\eta \mu_{8 s+1}$ where $\eta$ is the generator of $\Pi_{1}^{s}$.

Therefore we have the following diagram.

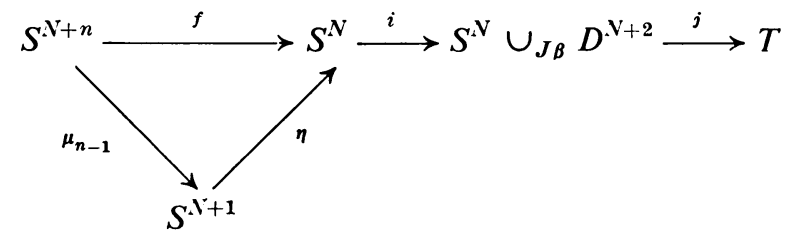

Since $J \beta=\eta, i \circ \eta \simeq *$, and $j \circ i \circ f \simeq *$. Thus, $k_{*} \mu_{n}=0$.

Remark. The lemma remains true if $\Pi_{1} M \neq(1)$ and $\Pi_{2}(M) \neq 0$ but $H_{2} \Pi_{1}$, the second homology group of $\Pi_{1}$, vanishes. This is enough to 
guarantee the existence of an imbedded $S^{2} \subset M$ in each homology class of $\mathrm{H}_{2} \mathrm{M}$.

Proof of the "only if" part of the main theorem. Let $\Sigma^{n}$ be a homotopy sphere represented by $\mu_{n}$, by the Thom-Pontriagin construction. The results of [2] imply that $\Sigma^{n}$ does not bound a spin manifold (see also [9]) and $\Sigma^{n} \notin k_{n}$. By the last lemma and previous corollary there exists $\Sigma_{1}^{n} \in \theta^{n}(\partial \pi)$ so that $\Sigma^{n} \# \Sigma_{1}^{n} \in I\left(M^{n}\right)$. But for $n$ even, $\theta^{n}(\partial \pi)=0 \quad[6]$; and so $\Sigma^{n} \in I\left(M^{n}\right.$.).

This completes the proof of the theorem.

\section{BIBLIOGRAPHY}

1. J. F. Adams, On the groups $J(X)$. IV, Topology 5 (1966), 21-71. MR 33 \#6628.

2. D. W. Anderson, E. M. Brown, Jr. and F. P. Peterson, The structure of the spin cobordism ring, Ann. of Math. (2) 86 (1967), 271-298. MR 36 \#2160.

3. G. E. Bredon and A. Kosinski, Vector field on $\pi$-manifolds, Ann. of Math. (2) 84 (1966), 85-90. MR 34 \#823.

4. A. Brender, Inertial and bordism properties of spheres, Proc. Amer. Math. Soc. 27 (1971), 209-212. MR 42 \#2494.

5. E. H. Brown and B. Steer, A note on Stiefel manifolds, Amer. J. Math. 87 (1965), 215-217. MR 30 \#5322.

6. M. Kervaire and J. Milnor, Group of homotopy spheres. I, Ann. of Math. (2) 77 (1963), 504-537. MR 26 \#5584.

7. M. Kervaire, A note on obstructions and characteristic classes, Amer. J. Math. 81 (1959), 773-784. MR 21 \#6585.

8. A. Kosinski, On the inertia group of $\pi$-manifolds, Amer. J. Math. 89 (1967), 227-248. MR 35 \#4936.

9. A. G. Naoum, On the groups of inertia of smooth manifolds, Ph.D. Dissertation, New York University, 1968.

10. S. P. Novikov, Homotopically equivalent smooth manifolds. I, Izv. Akad. Nauk SSSR Ser. Mat. 28 (1964), 365-475; English transl., Amer. Math. Soc. Transl. (2) 48 (1965), 271-396. MR 28 \#5445.

Department of Mathematics, College of Science, University of Baghdad, BAGHDAD, IRAQ 